Research Paper

\title{
Overexpression of PAK1 Correlates with Aberrant Expression of EMT Markers and Poor Prognosis in Non-Small Cell Lung Cancer
}

\author{
Zhiying Yang ${ }^{*}$, Heran Wang ${ }^{2 *}$, Longzheng $\mathrm{Xia}^{2}$, Linda Oyang${ }^{2}$, Yujuan Zhou ${ }^{2}$, Baihua Zhang', Xiaoyan \\ $\mathrm{Chen}^{2}$, Xia Luo ${ }^{2}$, Qianjin Liao $^{2 \bowtie}$, Jianping Liang ${ }^{2 \bowtie}$ \\ 1. Department of Histology and Embryology, Medical College, Hunan normal University, Changsha 410013, PR China; \\ 2. Key Laboratory of Translational Radiation Oncology, Hunan Province, Hunan Cancer Hospital and The Affiliated Cancer Hospital of Xiangya School of \\ Medicine, Central South University, 283 Tongzipo Road, Changsha 410013, Hunan, China. \\ * These authors contributed equally to this work. \\ $\square$ Corresponding authors: Qianjin Liao and Jianping Liang, Hunan Cancer Hospital and The Affiliated Cancer Hospital of Xiangya School of Medicine, Central \\ South University, 283 Tongzipo Road, Changsha 410013, Hunan, China. Tel: 86-731-88651680; Fax: 86-731-88651999. Email: liaoqianjin@hnszlyy.com, \\ liangiianping@hnszlyy.com \\ (c) Ivyspring International Publisher. This is an open access article distributed under the terms of the Creative Commons Attribution (CC BY-NC) license \\ (https://creativecommons.org/licenses/by-nc/4.0/). See http://ivyspring.com/terms for full terms and conditions.
}

Received: 2016.11.29; Accepted: 2017.03.06; Published: 2017.06.01

\begin{abstract}
Objective: p21-activated kinases (PAKs) are serine/threonine protein kinases. PAK1 and epithelial-mesenchymal transition (EMT) are key therapeutic targets in cancer. The clinical significance of PAK1 and its potential association with EMT phenotype in non-small cell lung cancer (NSCLC) was investigated.

Methods: Immunohistochemistry was used to detect the expression of PAK1, and mesenchymal and epithelial markers (vimentin, N-cadherin, and E-cadherin) in 186 cases of NSCLC tissues and 50 cases of tumor-adjacent normal tissues. The correlation of PAK1 with the clinicopathological characteristics, prognosis, and mesenchymal and epithelial markers in NSCLC were analyzed.

Results: Compared with the non-tumor tissues, PAK1, vimentin, and $\mathrm{N}$-cadherin levels were markedly elevated in NSCLC tissues, whereas the E-cadherin levels were significantly decreased $(P<0.05)$. The aberrant expression of PAK1 was significantly associated with TNM stage and metastasis $(P<0.001)$. Patients who displayed high expression of PAK1 may achieve a poorer progression-free survival (PFS) and overall survival (OS), compared to those with low expression of PAK1 ( $P=0.001$ and $P<0.001)$. Univariate and multivariate analysis showed that high expression of PAK 1 was an independent predictor of poor prognosis [hazard ratio $(\mathrm{HR})=2.121, \mathrm{P}<0.001, \mathrm{HR}=1.928, \mathrm{P}=0.001$, respectively]. In addition, significant correlations were observed between the EMT markers and OS or PFS $(P<0.01)$. Interestingly, PAK1 expression was positively correlated with vimentin and $\mathrm{N}$-cadherin levels $(r=0.473, \mathrm{P}<0.001$; $r=0.526, P<0.001$, respectively) and negatively correlated with E-cadherin levels $(r=-0.463, P<0.001)$ in NSCLC tissues.

Conclusion: PAK1 may promote NSCLC progression and metastasis through EMT, thereby exhibiting the potential of an efficient prognostic predictor in NSCLC patients.
\end{abstract}

Key words: PAK1; non-small cell lung cancer; epithelial-mesenchymal transition; prognosis; metastasis.

\section{Introduction}

Lung cancer is the leading cause of cancer-related deaths ${ }^{[1]}$, and approximately $85 \%$ of all the lung cancer cases are non-small-cell lung cancer (NSCLC), including large cell carcinoma, adenocarcinoma, squamous cell carcinoma, and bronchioloalveolar carcinoma[2]. Although recent advances are opted for in the clinical treatment, relapse within 2 years continues to prevail in approximately $65 \%$ NSCLC patients suitable for surgicalprocedures, and subsequently, succumb to 
mortality due to the metastatic spread[3]. However, the mechanism underlying the relapse and metastasis of NSCLC is yet unclear. Thus, the elucidation of the molecular mechanisms would facilitate the development of treatment strategies for NSCLC, specifically targeting the biological markers of metastases and tumor progression.

Epithelial-mesenchymal transition (EMT) is a molecular process, which allows the transformation of epithelial cells into a plastic and motile state with a mesenchymal phenotype. This characteristic is accompanied by the modifications in adhesion, morphology, cellular architecture, migration potential $[4,5]$, and altered expression of several genes involved in these processes. EMT is commonly characterized by the downregulation of E-cadherin (a critical cell-to-cell adhesion molecule), which is a well-established epithelial marker, and the upregulation of vimentin (a critical role in cell migration) and $\mathrm{N}$-cadherin (involved in a process known as cadherin switching) $\left.{ }^{[4,6}, 7\right]$. These features subsequently lead to the cytoskeletal reorganization, thereby further promoting EMT. Several studies have shown that EMT was implicated as a critical process in the tumor invasion and metastasis of various malignant tumors, such as breast ${ }^{[8]}$, colorectal[9], prostatic cancer[10], and lung cancer ${ }^{[11]}$. Moreover, EMT has been associated with the escape from apoptosis, radioresistance, chemoresistance, stemness, and poor prognosis ${ }^{12,13]}$. However, the underlying mechanism are yet to be elucidated.

A multitude of signaling pathways, such as TGF- $\beta /$ Smad, Ras/MAPK, Notch, and $W n t / \beta$-catenin regulate the EMT [14]. Recently, extensive studies involving Rac GTP enzyme (Rho protein family member) in the regulation of EMT in tumor cells have been carried out. This enzyme is demonstrated to play a key role in regulating adhesion, spreading, migration, and division and also involved in all the stages of cancer progression [15, 16]. p21-activated kinases (PAKs) are serine/threonine protein kinases and characterized as the downstream effectors of the Rho family small GTPases, Cdc42 and Rac1, the essential regulators of cell migration and invasion processes involved in cancer metastasis [17-19]. Therefore, it is common that PAKs serve as crucial mediators for the regulation of cytoskeletal remodeling, cell motility, morphology, and cell proliferation, thereby contributing towards cancer initiation, progression, and metastasis ${ }^{[17]}$. As a member of PAK family, PAK1 has been implicated in extracellular signaling that modulates cell polarity, invasion, and actin cytoskeleton organization[18]. PAK1 is over-expressed in various malignancies such as ovarian, breast, lung, and bladder cancers, and is closely associated with tumor invasion[20, 21]. The PAK1 expression level is positively correlated with tumor progression, metastases, and poor prognosis of specific carcinomas ${ }^{[20,22,23]}$. The inhibition of PAK1 expression or disruption of its function may significantly inhibit cancer cell proliferation and metastasis $[18,19,24,25]$. The gene amplification and overexpression of PAK1 could be significantly detected in the cytoplasm and nucleus of cells from NSCLC patients by genomic analysis. The overexpression or activation PAK1 distinctly promoted cell motility and invasiveness of NSCLC cells while selective inhibition targeting PAK1 could negatively regulate the cell proliferation both in vitro and in vivo[18,26]. In addition, other reports stated that activated PAK1 can contribute to therapeutic resistance $[27,28]$. Moreover, Yang et al. revealed that PAK1 was overexpressed in radioresistant NSCLC cells exposed to ionizing radiation (IR) ${ }^{[28]}$. Furthermore, the study also demonstrated that PAK1 tyrosine phosphorylation was required to induce EMT and radioresistance in lung cancer cells, which indicated PAK1 as a critical factor for conferring radioresistance ${ }^{[26]}$. However, the clinical and functional roles of PAK1 and the correlation of PAK1 and EMT markers throughout lung cancer initiation and progression remain obscure. Thus, we analyzed the clinical significance of PAK1 and investigated its probable association with EMT phenotype in NSCLC. In the present study, we found that the expression of PAK1 and EMT markers was significantly abnormal in NSCLC tissues, which was closely associated with TNM stage, metastasis, and prognosis. Moreover, we also inferred that PAK1 might be associated with EMT phenotype in NSCLC, therefore, promoting NSCLC invasion and metastasis resulting in poor prognosis.

\section{Materials and Methods}

\section{Tumor samples}

Lung cancer samples (186 cases) and para-carcinoma tissues (50 cases), obtained from Hunan Cancer Hospital/The Affiliated Cancer Hospital of Xiangya School of Medicine, Central South University (China) from 2002 to 2004, were used in the present retrospective study. All the patients diagnosed with NSCLC with complete medical records and adequate paraffin-embedded tissue blocks were eligible for participation in the study. Due to financial or other reasons, all the patients were not subjected to postoperative therapy (postoperative routine chemotherapy or radiotherapy). The follow-up duration was from 1.5 to 180 months, and the average was 45.8 months. The clinicopathological characteristicsare listed in Table 1. 
Table 1. Relationship between PAK1 expression and clinical pathology

\begin{tabular}{|c|c|c|c|c|}
\hline \multirow[t]{2}{*}{ Variable } & \multirow[t]{2}{*}{$\mathrm{N}$} & \multicolumn{3}{|l|}{ PAK1 } \\
\hline & & High & Low & $P$-value \\
\hline Age (years) & & 136 & 50 & \\
\hline$\leq 60$ & 123 & 90 & 33 & \\
\hline$>60$ & 63 & 46 & 17 & 0.982 \\
\hline \multicolumn{5}{|l|}{ Gender } \\
\hline male & 172 & 127 & 45 & 0.438 \\
\hline female & 14 & 9 & 5 & \\
\hline \multicolumn{5}{|l|}{ Smoking } \\
\hline Yes & 154 & 115 & 39 & 0.293 \\
\hline No & 32 & 21 & 11 & \\
\hline \multicolumn{5}{|l|}{ Histology } \\
\hline $\begin{array}{l}\text { Squamous cell } \\
\text { carcinoma }\end{array}$ & 133 & 95 & 38 & \multirow[t]{3}{*}{0.630} \\
\hline Adenocarcinoma & 30 & 24 & 6 & \\
\hline others & 23 & 17 & 6 & \\
\hline \multicolumn{5}{|l|}{ Histology grade } \\
\hline Well & 17 & 11 & 6 & \multirow{3}{*}{0.164} \\
\hline Moderate & 144 & 103 & 41 & \\
\hline $\begin{array}{l}\text { Poorly and Unknown } \\
\text { TNM stage }\end{array}$ & 25 & 22 & 3 & \\
\hline I & 21 & 6 & 15 & \multirow{4}{*}{0.000} \\
\hline II & 68 & 55 & 13 & \\
\hline III & 82 & 63 & 19 & \\
\hline IV & 15 & 12 & 3 & \\
\hline Metastasis & 134 & 108 & 26 & \\
\hline No metastasis & 52 & 28 & 24 & 0.000 \\
\hline
\end{tabular}

\section{Immunohistochemistry (IHC)}

Immunohistochemistry was performed on paraffin-embedded tissue sections, using the standard streptavidin/peroxidase staining method with the IHC kit (Com Win Biotech Co., Ltd., Beijing, China) according to the manufacturer's instructions. Briefly, the slides were dried, dewaxed in xylol, and rehydrated using a decreasing alcohol gradient. After blocking of endogenous peroxidase with $3 \% \mathrm{H}_{2} \mathrm{O}_{2}$, antigen retrieval was performed in $10 \mathrm{mM}$ citrate buffer and washed in PBS for $5 \mathrm{~min}$. Subsequently, the slides were blocked with $2 \%$ goat serum for $10 \mathrm{~min}$, followed by incubation with the following antibodies: primary monoclonal rabbit anti-PAK1 (1:400), mouse anti-N-cadherin (1:500, Abcam, Cambridge, UK), mouse anti-E-cadherin, and mouse anti-vimentin (Maxin, Fuzhou, China), overnight at $4^{\circ} \mathrm{C}$. The normal rabbit IgG was used as a negative control. The specimens were color-developed with 3,3'-diaminobenzidine (DAB) and counterstained with hematoxylin. The staining was brown or tan and localized in the cytoplasm or nucleus.

\section{IHC evaluation}

A semiquantitative scoring criterion for IHC was used where in the staining intensity and positive areas were recorded as described previously ${ }^{[29]}$. Briefly, the percentage of positive cells was determined by counting 500 cells in five randomly selected fields/section. The percentage of reactivity was scored as follows: 0 (no positive tumor cells), 1 ( $<10 \%$ positive tumor cells), 2 (10-50\% positive tumor cells), and 3 ( $>50 \%$ positive tumor cells). The intensity of staining was scored on a 0-3 scale: 0 (no staining), 1 (weak staining), 2 (moderate staining), and 3 (strong staining). Next, the final score was obtained by multiplying the intensity and reactivity rates. The scores of $<4$ suggested low expressions, and the remaining were classified as high expression. The immunostaining results were substantiated independently by two pathologists blinded to the samples and results.

\section{Statistical analysis}

All analyses were performed using SPSS 15.0 software (SPSS, Chicago, IL, USA). The correlation between the expression of PAK1 and clinicopathological parameters was analyzed using the chi-square test. Spearman's rank test was used to determine the correlation between PAK1 and EMT markers. Survival was estimated by the Kaplan-Meier method and compared with the log-rank test. Univariate and multivariate analyses were conducted using Coxregression model after adjusting for the baseline characteristics. A $P$-value of $<0.05$ was considered statistically significant.

\section{Results}

\section{Expression of PAK1 in NSCLC and adjacent normal tissues}

IHC was used to estimate the expression of PAK1 in NSCLC and the adjacent normal tissues. PAK1 was positively expressed mainly in the cytoplasm, showing distinct pale brown staining. Representative images for immunostaining were illustrated in Figure 1. A total of $73.1 \%(136 / 186)$ tumor samples showed high expression, whereas only $30 \%(15 / 50)$ showed a high expression in the tissues adjacent to the lung cancer. PAK1 protein expression in the NSCLC tissue was significantly higher than in the adjacent tissue $\left(\chi^{2}=28.156, P<0.001\right)$.

\section{Association between PAK1 protein expression and clinicopathological parameters}

As demonstrated in Table 1, PAK1 protein expression was remarkably associated with the TNM stage $(P<0.001)$ and metastasis $(P<0.001)$. However, clear correlations were not found in other clinicopathological parameters, including age, sex, smoking, pathological phenotype, or degree of differentiation $(P>0.05)$. 


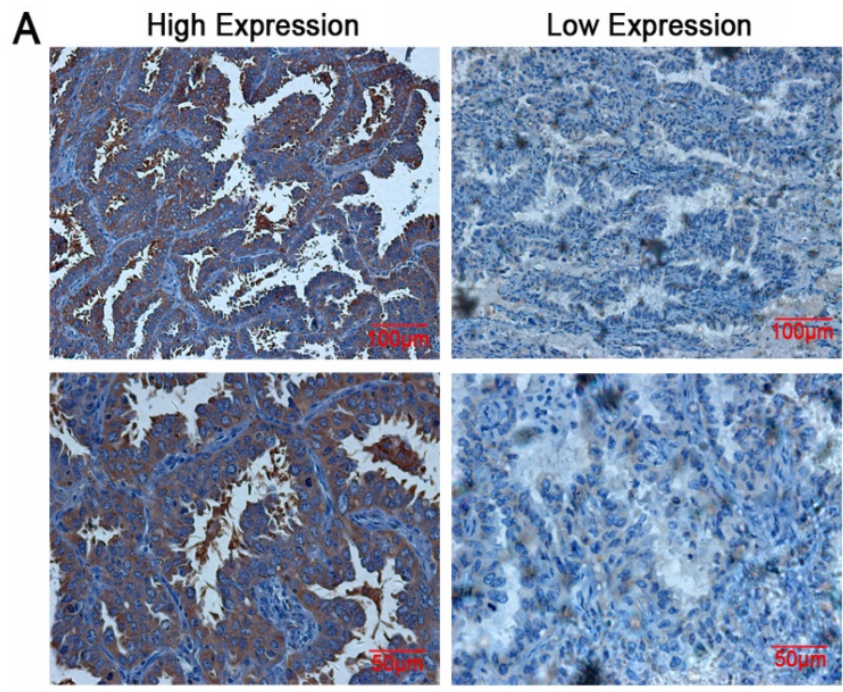

B

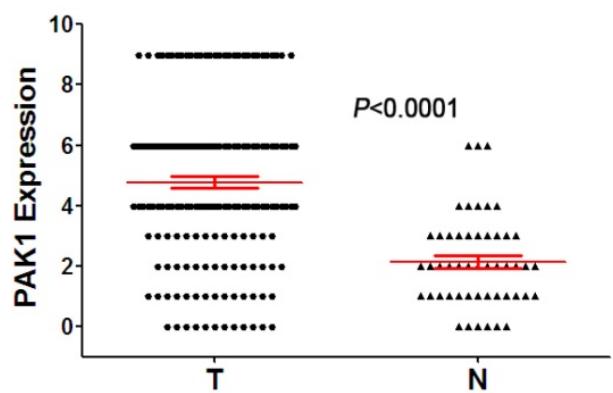

Figure 1. Expression of PAK1 in NSCLC tissues A: Representative IHC staining of PAK1 in NSCLC tissues (magnification $\times 200$, scale bars $100 \mu \mathrm{m}$; magnification $\times 400$, scale bars $50 \mu \mathrm{m}$ ); B: Scatter plot showed the expression levels of PAK1 in lung cancer $(\mathrm{T})$ and adjacent normal $(\mathrm{N})$ tissues.

\section{Prognostic significance of PAK1 in NSCLC patients}

Next, we examined the prognostic value of PAK1 protein expression. The association between PAK1 protein expression and clinical outcome in NSCLC patients was shown in Figure 2. The Kaplan-Meier survival analysis revealed that lung cancer patients with high protein expression of PAK1 resulted in $7.4 \%(10 / 136)$ progression-free survival

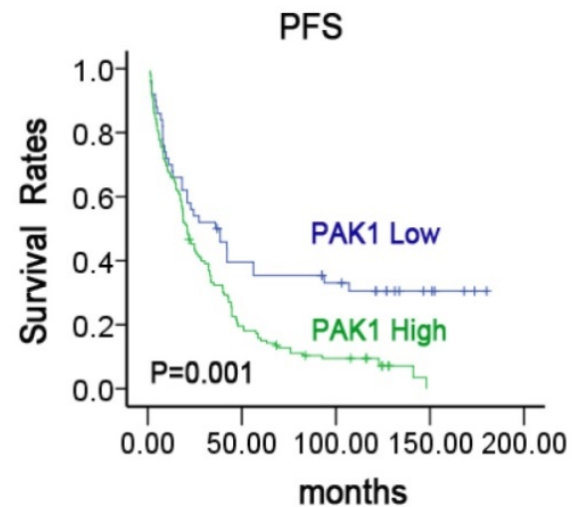

(PFS), as compared to $32.0 \%(16 / 50)$ with low expression $(P=0.001)$. A similar result was observed in the overall survival $(\mathrm{OS})$ analysis $(P<0.001)$. These findings suggested thatthe protein expression level of PAK1 can serve as an important and independent predictor in NSCLC patients.

Furthermore, Cox's univariate and multivariate analyses were applied for determining the independent prognostic factors. As listed in Table 2, PAK1 expression, clinical stage, and metastasis were significantly associated with $\mathrm{OS}(\mathrm{HR}=2.121, P<0.001$; $\mathrm{HR}=1.245, P=0.036$; $\mathrm{HR}=1.467, P=0.037)$ according to the univariate analysis, and other parameters showed no major prognostic value. The multivariate analysis showed that high expression of PAK1 exhibited a poor prognosis than those with low expression $(\mathrm{HR}=1.928, P=0.001)$. These findings suggested PAK1 as a potential prognostic biomarker of NSCLC.

\section{PAKI expression is associated with $\mathrm{N}$-cadherin, vimentin, and $\mathrm{E}$-cadherin in lung cancer tissues}

To determine the association between PAK1 expression and EMT, IHC was utilized to examine the expression of three widely acknowledged EMT markers (vimentin, N-cadherin, and E-cadherin). Compared with the normal tissues adjacent to lung cancer, the tumorous tissues significantly expressed vimentin and $\mathrm{N}$-cadherin, whereas the expression of E-cadherin was low (Figure 3A\& B). As demonstrated in Table 3, N-cadherin, vimentin, and E-cadherin expression showed significant correlation with lung cancer TNM stage and metastasis. E-cadherin expression showed significant correlation with smoking. Vimentin and E-cadherin expression was significantly correlated with differentiation of lung cancer. However, the EMT markers had no correlation with other clinicopathological parameters, including sex, age, pathological phenotype.

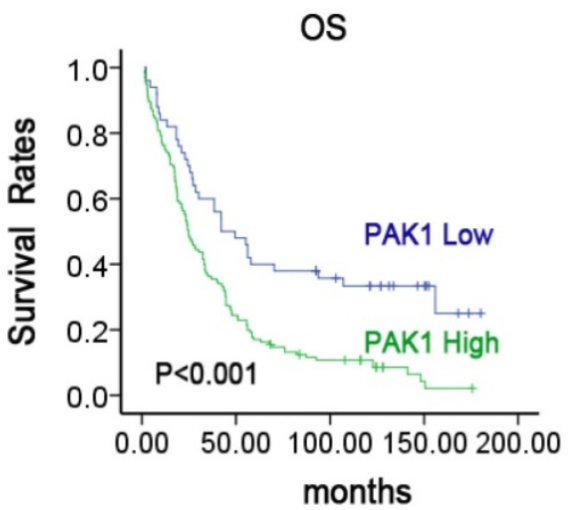

Figure 2. Survival analysis of patients with varying levels of PAK 1 expression in NSCLC Kaplan-Meier estimated PFS and OS in NSCLC patients based on PAK1 expression pattern. $P$-values were obtained using the log-rank test. 
Table 2. Univariate and multivariate analysis of factors associated with OS in NSCLC

\begin{tabular}{|c|c|c|c|c|c|c|}
\hline \multirow[t]{2}{*}{ Variables } & \multicolumn{3}{|c|}{ Univariate analysis } & \multicolumn{3}{|c|}{ Multivariate analysis } \\
\hline & $\overline{\mathrm{HR}}$ & $95 \% \mathrm{CI}$ & $P$ & HR & $95 \% \mathrm{CI}$ & $P$ \\
\hline Age $(\leq 60 />60$ years $)$ & 1.097 & $0.793-1.517$ & 0.575 & 1.297 & $0.913-1.842$ & 0.146 \\
\hline Gender (Female/Male) & 1.309 & $0.754-2.273$ & 0.339 & 1.441 & $0.639-3.249$ & 0.379 \\
\hline Smoking (Yes/No) & 0.955 & $0.631-1.445$ & 0.827 & 0.836 & $0.465-1.504$ & 0.152 \\
\hline Histology(Squamous Cell carcinoma/Adenocarcinoma/Others) & 0.882 & $0.640-1.216$ & 0.443 & 0.799 & $0.587-1.086$ & 0.152 \\
\hline Histology grade (Well/Moderate/Poorly and Unknown) & 1.419 & $0.889-2.034$ & 0.057 & 1.265 & $0.859-1.863$ & 0.233 \\
\hline Stage(I/II/III/IV) & 1.245 & $1.014-1.530$ & 0.036 & 1.131 & $0.898-1.425$ & 0.295 \\
\hline Metastasis(Yes/No) & 1.467 & $1.024-2.102$ & 0.037 & 1.351 & 0.893-2.045 & 0.155 \\
\hline PAK1(High/Low) & 2.121 & $1.441-3.120$ & 0.000 & 1.928 & $1.288-2.887$ & 0.001 \\
\hline
\end{tabular}

HR: Hazard ratio; CI: confidence interval.

A

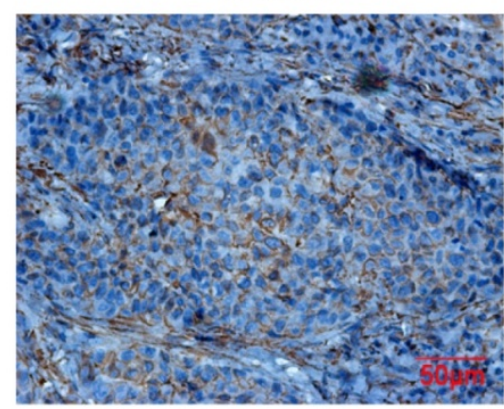

$\mathrm{N}$-cadherin

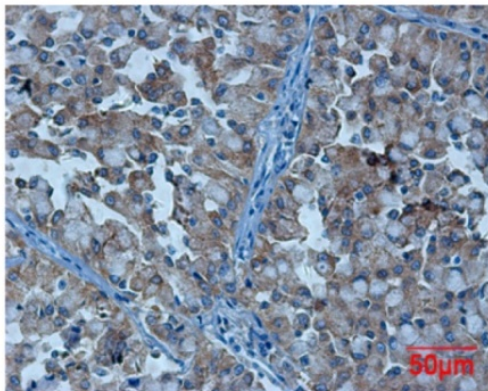

\section{E-cadherin}

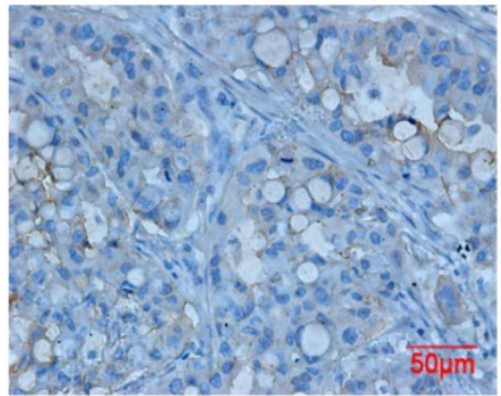

B

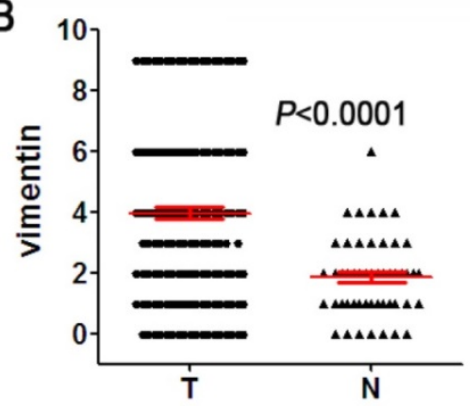

C
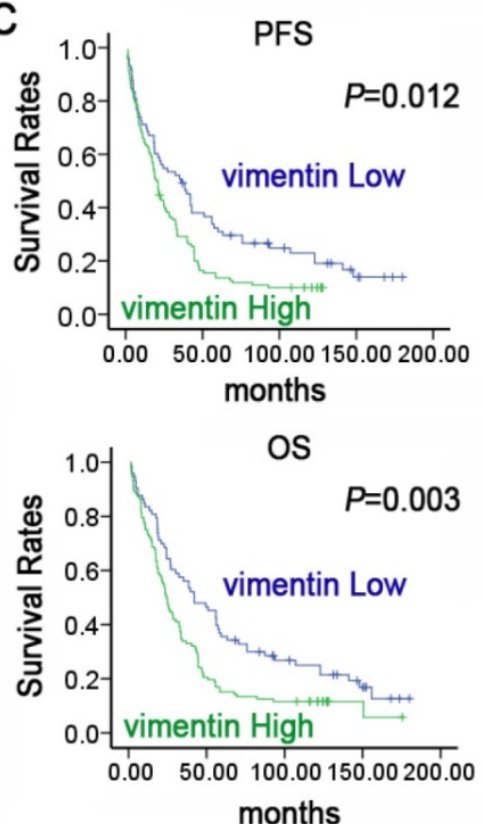
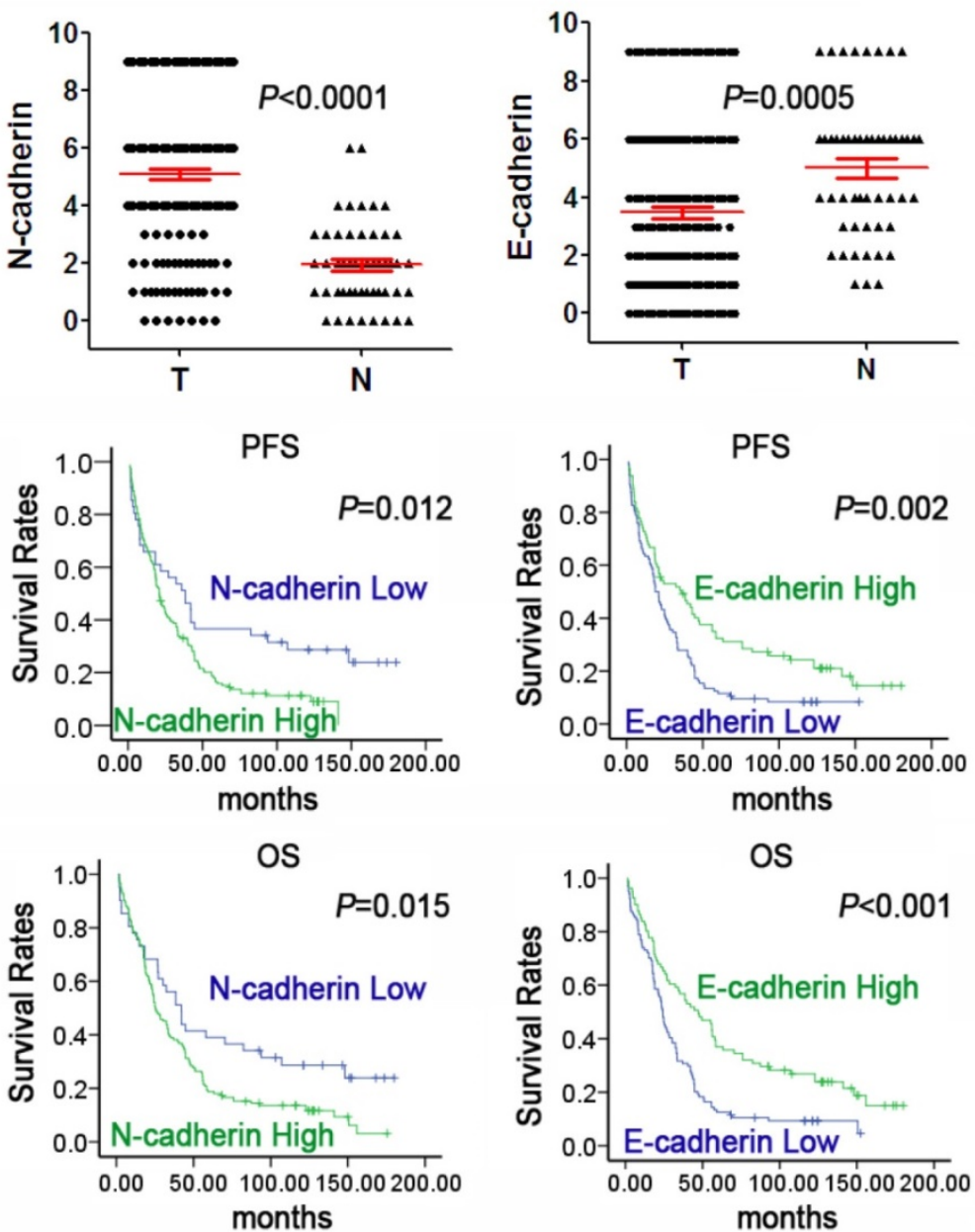

Figure 3. Expression of EMT markers and their prognostic role in NSCLC. A: Representative IHC staining of vimentin, N-cadherin, and E-cadherin in NSCLC tissues (magnification $\times 400$, scale bars $50 \mu \mathrm{m}$ ); B: Scatter plot showed the expression levels of vimentin, $N$-cadherin, and $E$-cadherin of lung cancer $(T)$ and adjacent normal(N) tissues. C: Kaplan-Meier survival curves of PFS and OS in NSCLC patients based on vimentin, N-cadherin, and E-cadherin expression levels. $P$-values were obtained using the log-rank test. 
Table 3. Relationship between EMT markers and clinicopathologic characteristics

\begin{tabular}{|c|c|c|c|c|c|c|c|c|c|c|}
\hline \multirow[t]{2}{*}{ Variable } & \multirow[t]{2}{*}{$\mathrm{N}$} & \multicolumn{3}{|l|}{ N-cad } & \multicolumn{3}{|l|}{ Vim } & \multicolumn{3}{|l|}{ E-cad } \\
\hline & & High & Low & $P$ Value & High & Low & $P$ Value & High & Low & $P$ Value \\
\hline \multicolumn{11}{|l|}{ Age (years) } \\
\hline$\leq 60$ & 123 & 95 & 28 & 0.740 & 73 & 50 & 0.736 & 53 & 70 & 0.702 \\
\hline$>60$ & 63 & 50 & 13 & & 39 & 24 & & 29 & 34 & \\
\hline \multicolumn{11}{|l|}{ Gender } \\
\hline male & 172 & 134 & 38 & 0.954 & 103 & 69 & 0.746 & 76 & 96 & 0.923 \\
\hline female & 14 & 11 & 3 & & 9 & 5 & & 6 & 8 & \\
\hline \multicolumn{11}{|l|}{ Smoking } \\
\hline Yes & 154 & 121 & 33 & 0.657 & 88 & 66 & 0.060 & 73 & 81 & 0.046 \\
\hline No & 32 & 24 & 8 & & 24 & 8 & & 9 & 23 & \\
\hline \multicolumn{11}{|l|}{ Histology } \\
\hline Squamous cell carcinoma & 133 & 106 & 27 & & 78 & 55 & & 59 & 74 & \\
\hline Adenocarcinoma & 30 & 21 & 9 & 0.511 & 17 & 13 & 0.351 & 17 & 13 & 0.084 \\
\hline others & 23 & 18 & 5 & & 17 & 6 & & 6 & 17 & \\
\hline \multicolumn{11}{|l|}{ Histology grade } \\
\hline Well & 17 & 16 & 1 & & 11 & 6 & & 10 & 7 & \\
\hline Moderate & 144 & 110 & 34 & 0.241 & 80 & 64 & 0.025 & 69 & 75 & 0.002 \\
\hline Poor and Unknown & 25 & 19 & 6 & & 21 & 4 & & 3 & 22 & \\
\hline \multicolumn{11}{|l|}{ TNM stage } \\
\hline I & 21 & 9 & 12 & & 4 & 17 & & 15 & 6 & \\
\hline II & 68 & 57 & 11 & 0.000 & 44 & 24 & 0.001 & 34 & 34 & 0.005 \\
\hline III & 82 & 65 & 17 & & 53 & 29 & & 30 & 52 & \\
\hline IV & 15 & 14 & 1 & & 11 & 4 & & 3 & 12 & \\
\hline Metastasis & 134 & 114 & 20 & & 95 & 39 & & 51 & 83 & \\
\hline No metastasis & 52 & 31 & 21 & 0.000 & 17 & 35 & 0.000 & 31 & 21 & 0.008 \\
\hline
\end{tabular}

Kaplan-Meier survival analysis showed that the low expression of vimentin and N-cadherin displayed a superior PFS and OS while low expression of E-cadherin exhibited a worse PFS and OS in lung cancer patients (Figure 3C). The results suggested that EMT markers might serve as potential prognostic markers of lung cancer. In addition, As shown in Table 4, Spearman's rank test showed a significantly positive association between PAK1 and vimentin or $\mathrm{N}$-cadherin protein expression in the tumor samples ( $\mathrm{r}=0.473, P<0.001 ; \mathrm{r}=0.526, P<0.001$, respectively); a remarkable negative correlation between PAK1 and E-cadherin expression was seen in carcinoma specimens $\quad(\mathrm{r}=-0.463, \quad P<0.001)$. These results suggested that PAK1 promoted the progression, invasion, and metastasis or relapse of lung cancer by inducing EMT.

Table 4. Correlation between PAKI expression and EMT markers

\begin{tabular}{|c|c|c|c|c|c|}
\hline \multirow[t]{2}{*}{ EMT markers } & \multirow[t]{2}{*}{$\mathrm{N}$} & \multicolumn{2}{|l|}{ PAK1 } & \multirow[t]{2}{*}{$\mathrm{r}$} & \multirow[t]{2}{*}{$P$} \\
\hline & & High expression & Low expression & & \\
\hline \multicolumn{6}{|l|}{ Vim } \\
\hline High expression & 112 & 101 & 11 & 0.473 & 0.000 \\
\hline Low expression & 74 & 35 & 39 & & \\
\hline \multicolumn{6}{|l|}{ N-cad } \\
\hline High expression & 145 & 124 & 21 & 0.526 & 0.000 \\
\hline Low expression & 41 & 12 & 29 & & \\
\hline \multicolumn{6}{|l|}{ E-cad } \\
\hline High expression & 82 & 41 & 41 & -0.463 & 0.000 \\
\hline Low expression & 104 & 95 & 9 & & \\
\hline
\end{tabular}

\section{Discussion}

An increasing number of studies have suggested that PAK1 may be involved in the tumor initiation, progression, and metastasis, which is amplified, overexpressed, or hyperactivated in a variety of cancers $[17,21,30]$. Gene amplification and overexpression of PAK1 can be detected in the cancer tissues. Moreover, PAK1 overexpression may be associated with the tumor formation, progression, and metastasis, as well as an efficient biomarker of poor prognosis $[18,22,26]$. Consistent with these findings, functional studies using transgenic mouse models have also demonstrated that the overexpression of PAK1 promoted the formation of preneoplastic lesions and malignant tumor ${ }^{[31,32]}$. However, the role of PAK1 in lung cancer remains inconclusive. A previous study demonstrated that PAK1 expression was upregulated in head and neck cancer, and was correlated with tumor invasiveness ${ }^{[33]}$. In addition, the elevated expression of PAK1 may act as an independent adverse prognostic biomarker for recurrence and survival in patients with non-metastatic clear cell renal cell carcinoma after nephrectomy ${ }^{[22]}$. In the current study, we found that PAK1 was highly expressed in lung cancer tissues, and the overexpression was positively correlated with the TNM stage, metastasis, and poorprognosis, suggesting a potential promotion of PAK1 in lung cancer initiation, progression, and metastasis. 
Several other studies suggested that metastasis is one of the most decisive factors influencing the patient prognosis. EMT has been known to broadly regulate the cancer metastasis ${ }^{[12,34]}$, which is one of the pivotal determining factors affecting patient prognosis[35]. Therefore, we also detected the protein expression of EMT markers (vimentin, N-cadherin, and E-cadherin) in lung cancer tissues by IHC and analyzed their potential association with PAK1. The results showed that the abnormal expression of vimentin, N-cadherin, and E-cadherin was closely related to TNM stage, metastasis and prognosis. Herein, for the first time, we found that PAK1 expression in lung cancer specimens was positively correlated with vimentin and $\mathrm{N}$-cadherin expression, whereas it was negatively correlated with E-cadherin expression, suggesting a potential involvement of PAK1 in EMT phenotype. Similarly, the previous in vitro and in vivo studies has demonstrated that the correlation of PAK1 and EMT-related proteins in various cancers. Jagadeeshan et al. found that PAK1 modulation regulates EMT causing significant alteration in the tumor microenvironment leading to the activation of pancreatic stellate cells (PSCs), which in turn contribute to Gemcitabine resistance ${ }^{[25]}$. Kim et al. showed that PAK1 tyrosine phosphorylation was required to induce EMT and radioresistance in lung cancer cells ${ }^{[26]}$. Therefore, it may be speculated that PAK1 may be involved in the development of lung cancer at least partially through involvement in EMT. However, to investigate the specific regulatory role of PAK1 in EMT, subsequent in vitro and in vivo assays are essential.

The PAK1 expression is upregulated in multiple cancers and is correlated with tumor invasiveness and therapeutic resistance ${ }^{[33,36]}$. The reduction of PAK1 expression by shRNA knock-down inhibited the proliferation of PANC-1 and MiaPaCa-2 pancreatic cancer cell lines, also enhanced the sensitivity of these cells to gemcitabine ${ }^{[37]}$. Similar results were obtained in the case of the prostatetumor ${ }^{[38]}$ and other tumors ${ }^{[18]}$. Recently, some studies have shown that the inhibition of PAK1 expression or activation using small chemical molecules, allosteric kinase inhibitor, microRNAs, or peptides derived from the autoinhibitory domain of PAK1 could significantly inhibit various cancer cells' proliferation and metastasis $[19,20,24]$. For instance, selective PAK1 inhibition using allosteric kinase inhibitor, IPA-3, which binds covalently to the PAK1 regulatory domain, was associated with decreased cellular survival and proliferation in squamous non-small cell lung carcinomas and breast cancers $[18,20]$. Therefore, with respect to PAK1, the protein overexpression was associated with lung cancer progression and metastasis, suggesting that targeting the PAK1 signal with PAK1 inhibitor might be an efficient strategy for lung cancer patients. Nevertheless, achieving this goal in a clinical setting continues to remain a challenge for bench researcher. Also, the mechanisms underlying the anticancer therapy necessitate further elucidation.

In summary, these impressive amounts of evidence suggested that PAK1 level was associated with cancer progression and metastasis. The overexpression of PAK1 correlated with the aberrant expression of EMT markers and poor clinical prognosis in NSCLC. Thus, PAK1 may be a potential therapeutic target and prognostic predictor in NSCLC patients in the forthcoming future. However, the specific PAK1-mediated molecular mechanisms that regulate the progression and metastasis of NSCLC should be investigated further in cellular and animal models.

\section{Abbreviations}

NSCLC: non-small cell lung cancer, EMT: epithelial-mesenchymal transition, PAKs: p21-activated kinases, PAK1: p21-activated kinase 1, IHC: Immunohistochemistry, PFS: progression-free survival, OS: overall survival.

\section{Acknowledgement}

This work was supported in part by grants from the following sources: the National Natural Science Foundation of China (81472595, 81402006); the Research Project of Health and Family Planning Commission of Hunan Province (B2016045); the Natural Science Foundation of Hunan Province (2015JJ2094); Development and Reform Commission of Hunan Province.

\section{Ethics Committee Approval and Patient Consent}

This study was approved by the Joint Ethics Committee of the Hunan Cancer Hospital and The Affiliated Cancer Hospital of Xiangya School of Medicine, Central South University in China. All the individuals participating in this project signed informed consent.

\section{Competing Interests}

The authors have declared that no competing interest exists.

\section{References}

1. Chen W, Zheng R, Baade PD, et al. Cancer statistics in China, 2015. CA Cancer J Clin. 2016; 66: 115-32.

2. Torre LA, Siegel RL, Jemal A. Lung Cancer Statistics. Adv Exp Med Biol. 2016; 893: 1-19.

3. Miller KD, Siegel RL, Lin CC, et al. Cancer treatment and survivorship statistics, 2016. CA Cancer J Clin. 2016; 66: 271-89. 
4. Cappellesso R, Marioni G, Crescenzi M, et al. The prognostic role of the epithelial-mesenchymal transition markers E-cadherin and Slug in laryngeal squamous cell carcinoma. Histopathology. 2015; 67: 491-500.

5. Thiery JP, Acloque H, Huang RY, et al. Epithelial-mesenchymal transitions in development and disease. Cell. 2009; 139: 871-90.

6. Seton-Rogers S. Epithelial-mesenchymal transition: Untangling EMT's functions. Nat Rev Cancer. 2016; 16: 1.

7. Zhou L, Yu L, Wu S, et al. Clinicopathological significance of KAI1 expression and epithelial-mesenchymal transition in non-small cell lung cancer. World J Surg Oncol. 2015; 13: 234.

8. Felipe Lima J, Nofech-Mozes S, Bayani J, et al. EMT in Breast Carcinoma-A Review. J Clin Med. 2016; 5.

9. Cao H, Xu E, Liu H, et al. Epithelial-mesenchymal transition in colorectal cancer metastasis: A system review. Pathol Res Pract. 2015; 211: 557-69.

10. Kanayama H. [Mechanism of invasion and metastasis of prostate cancer: over view]. Nihon Rinsho. 2016; 74 Suppl 3: 129-34.

11. Mittal V. Epithelial Mesenchymal Transition in Aggressive Lung Cancers. Adv Exp Med Biol. 2016; 890: 37-56.

12. Smith BN, Bhowmick NA. Role of EMT in Metastasis and Therapy Resistance. J Clin Med. 2016; 5.

13. Moncharmont C, Levy A, Guy JB, et al. Radiation-enhanced cell migration/invasion process: a review. Crit Rev Oncol Hematol. 2014; 92: 133-42.

14. Nieto MA, Huang RY, Jackson RA, et al. Emt: 2016. Cell. 2016; 166: 21-45.

15. Xiang S, Li M, Xie X, et al. Rapamycin inhibits epithelial-to-mesenchymal transition of peritoneal mesothelium cells through regulation of Rho GTPases. FEBS J. 2016; 283: 2309-25.

16. Su B, Su J, Zeng Y, et al. Diallyl disulfide suppresses epithelial-mesenchymal transition, invasion and proliferation by downregulation of LIMK1 in gastric cancer. Oncotarget. 2016; 7: 10498-512.

17. Tse EY, Ching YP. The role of p21-activated kinases in hepatocellular carcinoma metastasis. J Mol Signal. 2014; 9: 7.

18. Ong CC, Jubb AM, Haverty PM, et al. Targeting p21-activated kinase 1 (PAK1) to induce apoptosis of tumor cells. Proc Natl Acad Sci U S A. 2011; 108: 7177-82.

19. Senapedis W, Crochiere M, Baloglu E, et al. Therapeutic Potential of Targeting PAK Signaling. Anticancer Agents Med Chem. 2016; 16: 75-88

20. Eswaran J, Li DQ, Shah A, et al. Molecular pathways: targeting p21-activated kinase 1 signaling in cancer--opportunities, challenges, and limitations. Clin Cancer Res. 2012; 18: 3743-9.

21. Dammann $\mathrm{K}$, Khare V, Harpain F, et al. PAK1 promotes intestinal tumor initiation. Cancer Prev Res (Phila). 2015; 8: 1093-101.

22. Feng Y, Fang S, Li M. Expression of P21-activated kinase 1 and cell division control protein 42 homolog correlates with clinicopathological features and prognosis in cervical carcinoma. J Obstet Gynaecol Res. 2016; 42: 860-9.

23. Zhu Y, Xu L, An H, et al. p21-activated kinase 1 predicts recurrence and survival in patients with non-metastatic clear cell renal cell carcinoma. Int J Urol. 2015; 22: 447-53.

24. Jiao D, Zhang XD. Myricetin suppresses p21-activated kinase 1 in human breast cancer MCF-7 cells through downstream signaling of the beta-catenin pathway. Oncol Rep. 2016; 36: 342-8.

25. Jagadeeshan S, Subramanian A, Tentu S, et al. P21-activated kinase 1 (Pak1) signaling influences therapeutic outcome in pancreatic cancer. Ann Oncol. 2016; 27: 1546-56.

26. Kim E, Youn $\mathrm{H}$, Kwon T, et al. PAK1 tyrosine phosphorylation is required to induce epithelial-mesenchymal transition and radioresistance in lung cancer cells. Cancer Res. 2014; 74: 5520-31.

27. Huynh N, Shulkes A, Baldwin G, et al. Up-regulation of stem cell markers by P21-activated kinase 1 contributes to 5-fluorouracil resistance of colorectal cancer. Cancer Biol Ther. 2016; 17: 813-23.

28. Yang HJ, Kim N, Seong KM, et al. Investigation of radiation-induced transcriptome profile of radioresistant non-small cell lung cancer A549 cells using RNA-seq. PLoS One. 2013; 8: e59319.

29. Zhou Y, Liao Q, Li X, et al. HYOU1, Regulated by LPLUNC1, Is Up-Regulated in Nasopharyngeal Carcinoma and Associated with Poor Prognosis. J Cancer. 2016; 7: 367-76.

30. Radu M, Semenova G, Kosoff R, et al. PAK signalling during the development and progression of cancer. Nat Rev Cancer. 2014; 14: 13-25.

31. Wang RA, Zhang H, Balasenthil S, et al. PAK1 hyperactivation is sufficient for mammary gland tumor formation. Oncogene. 2006; 25: 2931-6.

32. Arias-Romero LE, Villamar-Cruz O, Huang M, et al. Pak1 kinase links ErbB2 to beta-catenin in transformation of breast epithelial cells. Cancer Res. 2013; 73: 3671-82.

33. Park J, Kim JM, Park JK, et al. Association of p21-activated kinase-1 activity with aggressive tumor behavior and poor prognosis of head and neck cancer. Head Neck. 2015; 37: 953-63.

34. Diepenbruck M, Christofori G. Epithelial-mesenchymal transition (EMT) and metastasis: yes, no, maybe? Curr Opin Cell Biol. 2016; 43: 7-13.

35. Hanahan D, Weinberg RA. Hallmarks of cancer: the next generation. Cell. 2011; 144: 646-74.

36. Holm C, Rayala S, Jirstrom K, et al. Association between Pak1 expression and subcellular localization and tamoxifen resistance in breast cancer patients. J Natl Cancer Inst. 2006; 98: 671-80.
37. Yeo D, He H, Patel O, et al. FRAX597, a PAK1 inhibitor, synergistically reduces pancreatic cancer growth when combined with gemcitabine. BMC Cancer. 2016; 16: 24.

38. Goc A, Al-Azayzih A, Abdalla M, et al. P21 activated kinase-1 (Pak1) promotes prostate tumor growth and microinvasion via inhibition of transforming growth factor beta expression and enhanced matrix metalloproteinase 9 secretion. J Biol Chem. 2013; 288: 3025-35. 